\title{
New Diels-Alder Reactions of (-)-Thebaine and First X-Ray Crystallographic Structure Analyses of the Cycloadducts
}

\author{
Ulf Pindur*,a, Diana Keilhofer ${ }^{\mathrm{a}}$, Dieter Schollmeyer ${ }^{\mathrm{b}}$ \\ a Institut für Pharmazie, Fachbereich Chemie und Pharmazie der Universität, \\ D-55099 Mainz, Federal Republic of Germany \\ b Institut für Organische Chemie, Fachbereich Chemie und Pharmazie der Universität, \\ D-55099 Mainz, Federal Republic of Germany
}

Z. Naturforsch. 49b, 272-279 (1994); received October 25, 1993

(-)-Thebaine, Diels-Alder Reactions, X-Ray

Diels-Alder reactions of (-)-thebaine (1) as an electron-rich diene system with acceptorsubstituted ethenes gave rise to the cycloadducts $\mathbf{2 , 3}$, and $\mathbf{4}$ in high regio- and stereoselectivities. Structural analyses were performed by high resolution NMR spectroscopy and, for the first time in the thebaine cycloadduct series, by X-ray crystallographic structural analyses of the compounds $\mathbf{2}, \mathbf{3} \mathbf{a}$, and $\mathbf{3} \mathbf{b}$. In the reaction of (-)-thebaine (1) with an in situ generated aryne, the anellated azocine derivative 5 was formed.

Diels-Alder reactions of the opium alkaloid (-)-thebaine (1) as an electron-rich diene have been the subject of several investigations, in particular in the field of medicinal chemistry for the development of drugs with analgesic activities [1-5]. Recently, we have fulfilled the demand for more precise information on the stereochemistries of some reinvestigated and newly synthesized cycloadducts of thebaine by means of high resolution ${ }^{1} \mathrm{H}-\mathrm{NMR}$ spectroscopy [6]. It was concluded that the $\beta$-face endo approach [6] of the dienophile to the cyclohexadiene ring $\mathrm{C}$ in $\mathbf{1}$ is unambiguously favoured on steric and electronic grounds; moreover, in the latter case, the experimental results are fully compatible with frontier molecular orbital considerations [6]. In the context of our current investigations on the cycloaddition reactions of various electron-rich heterocycles [7], we are also continuing our studies of these special Diels-Alder reactions with (-)-thebaine (1), because the results will be of general interest for the future development of drugs.

In this paper, we describe some further DielsAlder reactions of (-)-thebaine (1) with selected carbodienophiles and report the first X-ray crystallographic structural analyses of Diels-Alder adducts of thebaine. These structural investigations will most certainly shed new light on the some-

\footnotetext{
* Reprint requests to Prof. Dr. U. Pindur.

Verlag der Zeitschrift für Naturforschung, D-72072 Tübingen

0932-0776/94/0200-0272/\$01.00/0
}

times rather unclear and less convincing configurational data given in the earlier reports $[1-4]$ and, last but not least, also provide incontrovertible sustenance for our recent, more detailed report [6].

\section{Results and Discussion}

\section{Synthetic aspects}

(-)-Thebaine (1) reacts with divinylsulfone regio- and stereoselectively to furnish the DielsAlder product 2 (63\% yield). No other isomeric cycloadducts were detected by TLC. However, this product was previously described in ref. [8], but no fully convincing data for the (absolute) stereochemistry of the newly formed stereocentres were given. The structure of $\mathbf{2}$ has now been unambiguously elucidated by NMR spectroscopy and X-ray crystallography (see below and Fig. 2). According to semiempirical quantum mechanical AM 1 calculations [9] (for details see [6]), the dienophile attacks the $\beta$ side of the diene system and the thus resulting endo-transition state is energetically favoured by $\mathrm{HOMO}($ diene)LUMO(dienophile) interactions involving secondary orbital overlap [10]. In further experiments, we examined the Diels-Alder reactivity of $\mathbf{1}$ towards some (E)- and (Z)-1,2-ethenedicarboxylates and found that 1 reacts with dimethyl and diethyl fumarate to give the cycloadducts $\mathbf{3} \mathbf{a}$ and $\mathbf{3 b}$ stereoselectively (by TLC) in 48 and $52 \%$ yields, respectively. In these cases also, the stereochemistries were clarified by NMR spectroscopy and X-ray crystallography (see Fig. 3). In summary, the three 
performed X-ray structural analyses of products $\mathbf{2}$, $\mathbf{3 a}$, and $\mathbf{3 b}$ are unique in the Diels-Alder chemistry of thebaine and further substantiate the structural investigations on the previous Diels-Alder adducts prepared from 1 [6].

It is known that $(Z)$-dialkyl 1,2-ethenedicarboxylates are, in general, less reactive than their (E)-counterparts [12]. Thus, in our hands, dimethyl maleate reacted with $\mathbf{1}$ to give a lower yield $(28 \%)$ of the cycloadduct 4 [11], albeit stereoselectively. The stereoselective Diels-Alder reactions of (-)-thebaine (1) with these dialkyl 1,2-ethenedicarboxylates suggest the exclusive participation of a concerted mechanism.

Acetylenic dienophiles react, in some cases, with (-)-thebaine (1) via a one-bond addition and subsequent piperidine ring enlargement reaction to

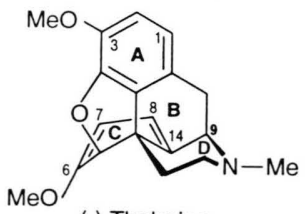

(-) Thebaine

1

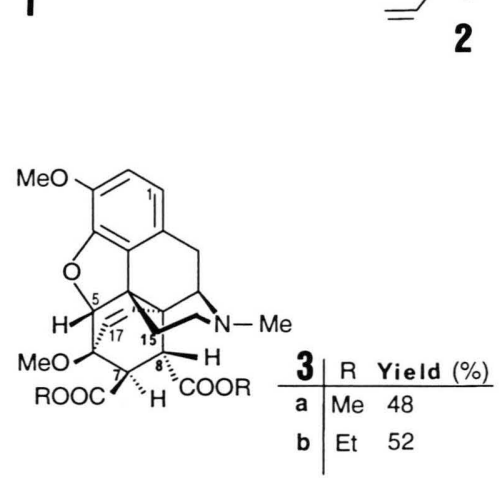

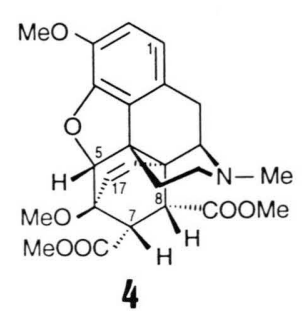

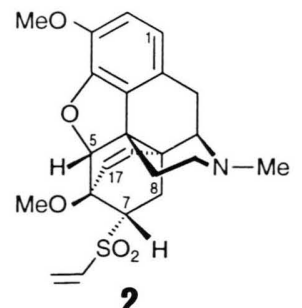

yield polycyclic azocine derivatives [13-15]. We have now found that an in situ generated aryne is also able to effect a ring enlargement reaction in $\mathbf{1 .}$ Thus, (-)-thebaine (1) reacted with benzyne, readily generated from diazotized anthranilic acid [16], to give rise to the hexacyclic benzo[ $b]$ naphtho[2,1$e$ ]azocine 5 , albeit only in $6 \%$ yield together with several other, less stable products. However, no normal Diels-Alder product could be isolated from crude reaction mixture. As a mechanistic rationale for this reaction, we propose a primary nucleophilic attack of the piperidine nitrogen atom at one aryne sp-centre [17]. The thus formed intermediate is then stabilized by heterolytic $\mathrm{C} 9-\mathrm{N}$ $\sigma$-bond cleavage which initiates the piperidine ring enlargement reaction (for an analogous mechanistic explanation, see ref. [14]).

\section{NMR spectroscopic investigations}

The ${ }^{1} \mathrm{H}$ and ${ }^{13} \mathrm{C}$ NMR spectroscopic data of the new Diels-Alder adducts are fully compatible with the recently reported data [6]. Typical for the stereochemistry of the Diels-Alder products is, for example, the $\mathrm{W}$-coupling of $\mathrm{H} 5$ with $\mathrm{H} 17$ (approx. $1.20 \mathrm{~Hz}$ ). Additionally, ${ }^{1} \mathrm{H},{ }^{1} \mathrm{H}$ NOE measurements are frequently useful to obtain more configura-

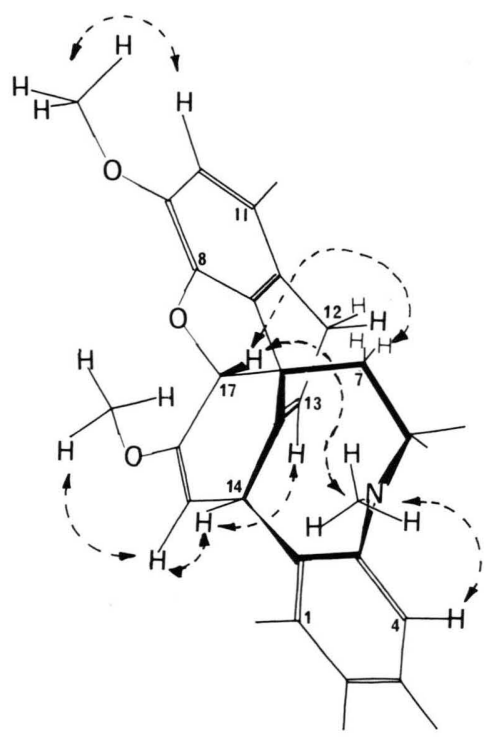

Fig. 1. ${ }^{1} \mathrm{H},{ }^{1} \mathrm{H}-\mathrm{Nuclear}$ Overhauser effects of compound 5 (400 MHz). An INDOR effect is registered between $\mathrm{H} 12$ and $\mathrm{H} 13$. The given geometry was established by MMX force field calculations and represents a local minimum [19]. 
Table I. Crystallographic data and structure determination details for $\mathbf{2 ,} \mathbf{3} \mathbf{a}$ and $\mathbf{3 b}$.

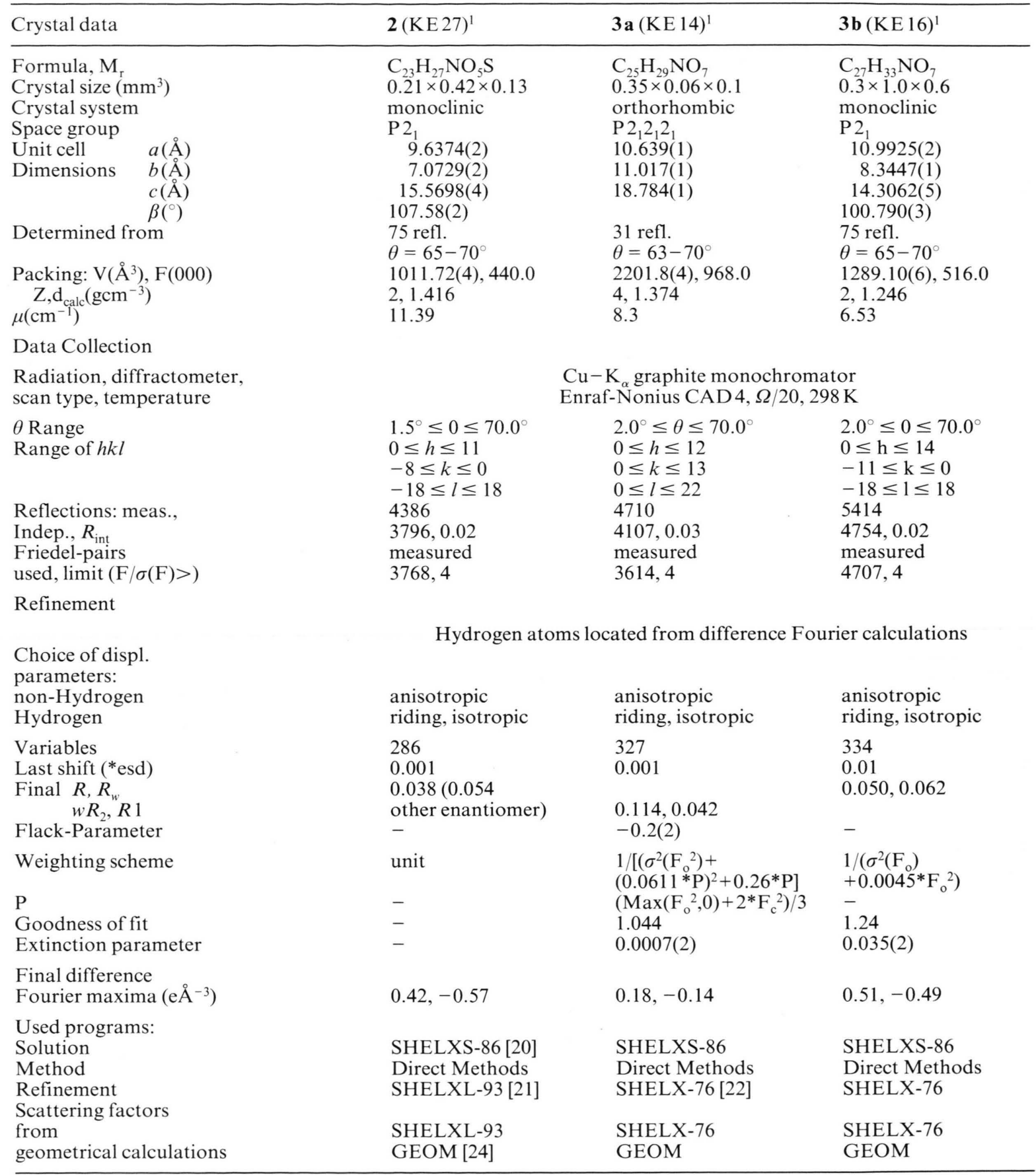

\footnotetext{
1 Substance code in supplemental material.
} 
tional details about the newly formed stereocentres. In the case of compound $\mathbf{3} \mathbf{a}$, interatomic distances were exemplarily analyzed by NOE experiments (steady state NOE) and proton $\mathrm{T}_{1}$ measurements [18]. On the basis of a calculated (fixed) $\mathrm{H}-\mathrm{H}$ reference distance (averaged distance $\mathrm{H} 5-\mathrm{H}, \mathrm{H} 15 \mathrm{a}, \mathrm{b}$ by MMX molecular mechanics method $=2.58 \AA[19])$, the configurations at C7 and $C 8$ were established. In summary, the relevance of more sophisticated NMR spectroscopic configuration analysis in the thebaine cycloadduct series is nicely illustrated by these studies in combination with the X-ray crystallographic results.

In the case of the hexacyclic product $\mathbf{5}$, firstly the diagnostically relevant ${ }^{1} \mathrm{H},{ }^{1} \mathrm{H}$ NOE's from $\mathrm{H} 17$ to $\mathrm{N} 5-\mathrm{CH}_{3}$ and vice versa (averaged $\mathrm{H}-\mathrm{H}$ distance of approx. $3.2 \AA$ according to MMX force field calculations [19], see also Fig. 1), in addition, a paramagnetic shift of the signal for $\mathrm{H} 17$ of approx $0.5 \mathrm{ppm}$ in comparison to the analogous shift in the thebaine cycloadducts and an $\mathrm{H}, \mathrm{H}-\mathrm{COSY}$ spectrum are highly indicative for the structure of 5 given.

\section{$X$-ray crystallographic structural analyses of $\mathbf{2 ,} \mathbf{3} \mathbf{a}$, and $\mathbf{3 b}$}

Experimental details for the structural analyses of compounds $\mathbf{2}, \mathbf{3} \mathbf{a}$, and $\mathbf{3} \mathbf{b}$ are listed in Table I. The investigated compounds all crystallized in non-centrosymmetric space groups, thus only one enantiomer is present in each case. As shown in Table II, the most important geometrical parameters for the compounds are all equal within the limits of accuracy and lie in the normal range. The phenyl ring is approximately planar, the five-membered ring $\mathrm{C} 4, \mathrm{C} 12, \mathrm{C} 13, \mathrm{C} 5, \mathrm{O} 1$ is twisted with $\mathrm{C} 5$ being above and $\mathrm{C} 13$ being below the least squares plane. As shown in Fig. 2, it can be concluded from the least squares calculations and from the values of the torsional angles that the cyclohexane ring has the boat conformation, while the piperidine ring takes up the chair conformation. The etheno bridge $\mathrm{C} 6-\mathrm{C} 17-\mathrm{C} 18-\mathrm{C} 14$ is nearly perpendicular to the least squares plane of the cyclohexane ring.

Further details of X-ray structural analyses can be obtained on request from the Fachinformationszentrum Karlsruhe, Gesellschaft für wissenschaftlich-technische Information $\mathrm{mbH}, \mathrm{D}-76344 \mathrm{Eg}$ -
genstein-Leopoldshafen, Germany, on quotation of the deposition number CSD-57719, the authors names, and the full journal citation.

Table II. Selected bond lengths for $\mathbf{2 , 3} \mathbf{3}$ and $\mathbf{3} \mathbf{b}$.

\begin{tabular}{|c|c|c|c|}
\hline Bond & 2 & $3 \mathbf{a}$ & $3 \mathbf{b}$ \\
\hline $\mathrm{O} 1-\mathrm{C}_{4}$ & $1.394(4)$ & $1.377(3)$ & $1.376(2)$ \\
\hline $\mathrm{O} 1-\mathrm{C} 5$ & $1.452(4)$ & $1.471(3)$ & $1.465(2)$ \\
\hline $\mathrm{N} 1-\mathrm{C} 9$ & $1.487(4)$ & $1.473(3)$ & $1.471(3)$ \\
\hline $\mathrm{N} 1-\mathrm{C} 16$ & $1.468(5)$ & $1.456(4)$ & $1.465(3)$ \\
\hline $\mathrm{C} 1-\mathrm{C} 2$ & $1.394(5)$ & $1.401(4)$ & $1.404(4)$ \\
\hline $\mathrm{C} 1-\mathrm{C}_{11}$ & $1.392(5)$ & $1.394(3)$ & $1.394(4)$ \\
\hline $\mathrm{C} 2-\mathrm{C} 3$ & $1.399(6)$ & $1.390(3)$ & $1.396(4)$ \\
\hline $\mathrm{C} 3-\mathrm{C} 4$ & $1.377(5)$ & $1.389(3)$ & $1.387(3)$ \\
\hline $\mathrm{C} 4-\mathrm{C} 12$ & $1.383(5)$ & $1.373(3)$ & $1.385(2)$ \\
\hline $\mathrm{C} 5-\mathrm{C} 6$ & $1.563(4)$ & $1.543(3)$ & $1.539(2)$ \\
\hline $\mathrm{C} 5-\mathrm{C} 13$ & $1.542(4)$ & $1.536(3)$ & $1.545(2)$ \\
\hline $\mathrm{C}_{6}-\mathrm{C} 7$ & $1.577(5)$ & $1.573(3)$ & $1.582(2)$ \\
\hline $\mathrm{C} 6-\mathrm{C} 17$ & $1.495(5)$ & $1.514(3)$ & $1.508(3)$ \\
\hline $\mathrm{C} 7-\mathrm{C} 8$ & $1.569(4)$ & $1.548(3)$ & $1.560(2)$ \\
\hline $\mathrm{C} 8-\mathrm{C} 14$ & $1.541(5)$ & $1.571(3)$ & $1.542(3)$ \\
\hline C $9-\mathrm{C} 10$ & $1.556(5)$ & $1.572(3)$ & $1.567(3)$ \\
\hline $\mathrm{C} 9-\mathrm{C} 14$ & $1.544(4)$ & $1.542(3)$ & $1.552(3)$ \\
\hline $\mathrm{C} 10-\mathrm{C} 11$ & $1.514(5)$ & $1.515(4)$ & $1.528(3)$ \\
\hline $\mathrm{C} 11-\mathrm{C} 12$ & $1.383(5)$ & $1.372(3)$ & $1.373(3)$ \\
\hline $\mathrm{C} 12-\mathrm{C} 13$ & $1.495(5)$ & $1.499(3)$ & $1.498(3)$ \\
\hline $\mathrm{C} 13-\mathrm{C} 14$ & $1.541(5)$ & $1.565(3)$ & $1.563(2)$ \\
\hline C $13-C 15$ & $1.545(5)$ & $1.537(3)$ & $1.539(3)$ \\
\hline $\mathrm{C} 14-\mathrm{C} 18$ & $1.520(5)$ & $1.510(3)$ & $1.515(3)$ \\
\hline C $15-C 16$ & $1.525(5)$ & $1.521(4)$ & $1.516(3)$ \\
\hline C $17-C 18$ & $1.317(4)$ & $1.321(3)$ & $1.332(3)$ \\
\hline
\end{tabular}

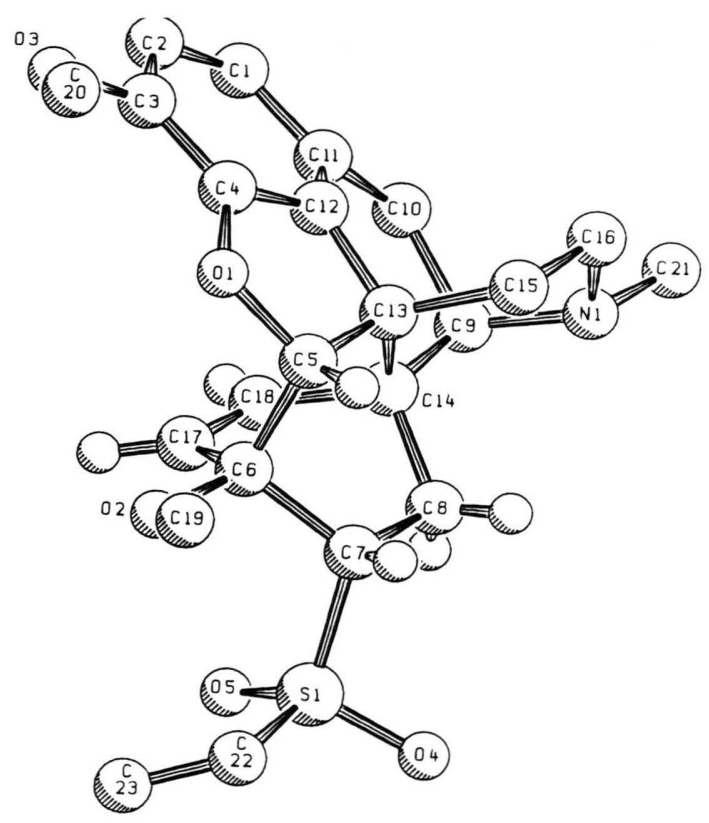

Fig. 2. X-ray struture of 2, SCHAKAL plot [23]. 

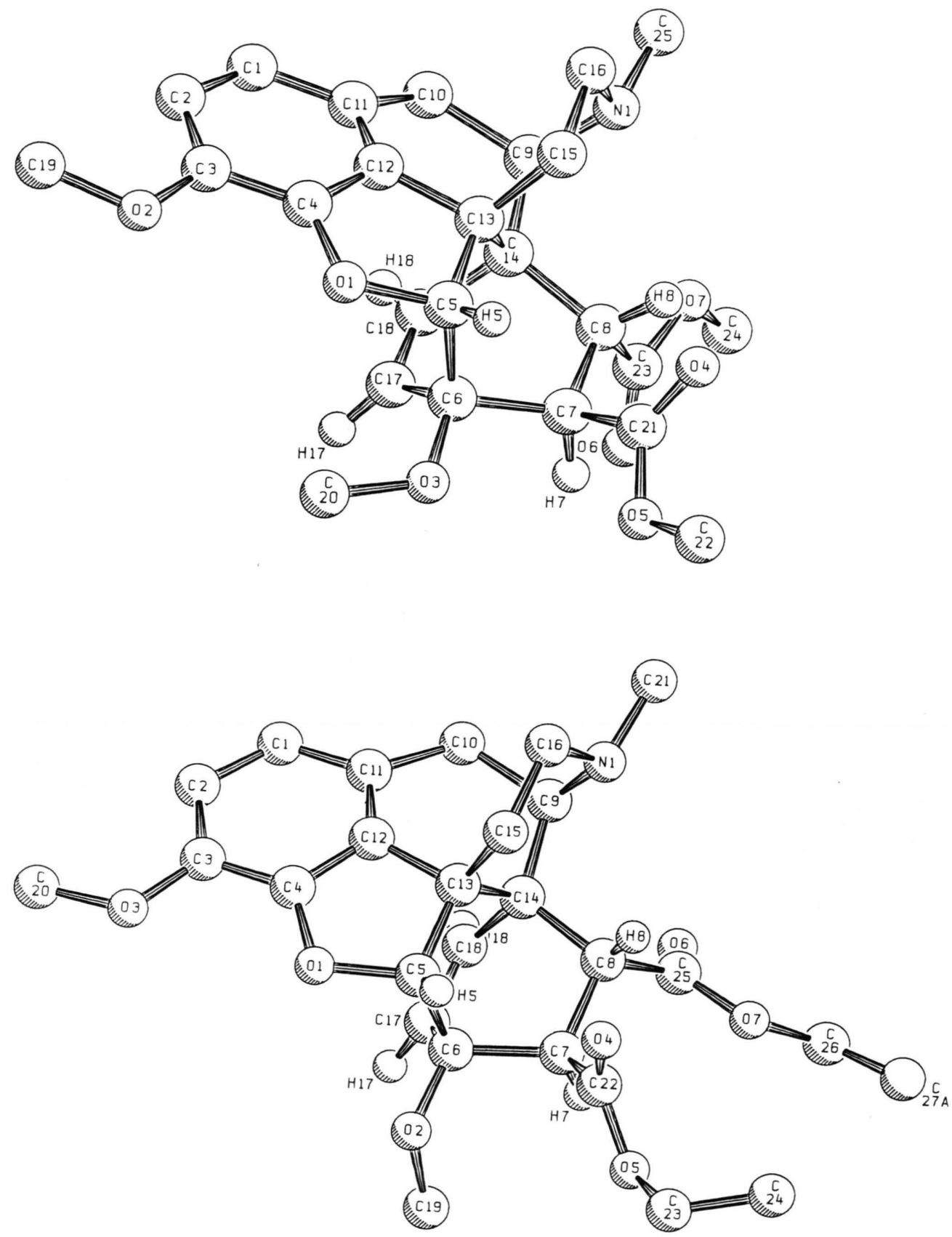

Fig. 3. X-ray structures of $\mathbf{3} \mathbf{a}$ (above) and $\mathbf{3 b}$ (below), SCHAKAL plot [23]. A disorder was found at C27. One of the possible orders was chosen. 


\section{Experimental}

${ }^{1} \mathrm{H}$ NMR spectra were recorded at 200 and 400 $\mathrm{MHz}$ with Bruker AC 200 and AMX 400 spectrometers. ${ }^{13} \mathrm{C}$ NMR spectra were measured at 50.3 $\mathrm{MHz}$ with a Bruker AC 200 instrument; $J$-modulated spin echo spectra were obtained in all cases $\left(\mathrm{C}_{\mathrm{p}}=\right.$ primary, $\mathrm{C}_{\mathrm{s}}=$ secondary, $\mathrm{C}_{\mathrm{t}}=$ tertiary, and $\mathrm{C}_{\mathrm{q}}=$ quaternary carbon atoms). The EI (70 $\mathrm{eV)}$ mass spectra were recorded on a Varian MAT 7 spectrometer. Elemental analyses were performed by using a Carlo Erba Strumentazione 1106 apparatus. Melting points were measured with an Electrothermal 8200 instrument. Flash chromatography was performed on Merck 60 silica gel (particle size: $0.040-0.063 \mathrm{~mm}$ ). The petroleum ether used had the boiling range $40-60{ }^{\circ} \mathrm{C}$.

All reactions were performed in highly pure, anhydrous solvents. The yields given refer to analytically pure compounds, some product loss occurred during chromatographic work-up.

\section{4,5 a-Epoxy-3,6-dimethoxy- $N$-methyl-7 a-vinyl- sulfonyl-6a, 14 a-endo-etheno-isomorphinane (2)}

(-)-Thebaine (1) (200 mg, $0.64 \mathrm{mmol})$ was dissolved in $20 \mathrm{ml}$ toluene. After addition of divinylsulfone $(75.6 \mathrm{mg}, 0.64 \mathrm{mmol})$, the mixture was stirred for $24 \mathrm{~h}$ at $20^{\circ} \mathrm{C}$. The formed precipitate was recrystallized four times from ethyl acetate/ petroleum ether. Yield $170 \mathrm{mg}(63 \%)$, m.p. $188-$ $190{ }^{\circ} \mathrm{C}$ (ethyl acetate, petroleum ether), 190$192{ }^{\circ} \mathrm{C}[8] .-{ }^{1} \mathrm{H}$ NMR $\left(200 \mathrm{MHz}, \mathrm{CDCl}_{3}\right): \delta=$ $1.81\left(\mathrm{~m}_{\mathrm{c}}, 4 \mathrm{H}\right), 2.33\left(\mathrm{~s}, 3 \mathrm{H}, \mathrm{NCH}_{3}\right), 2.43\left(\mathrm{~m}_{\mathrm{c}}, 3 \mathrm{H}\right)$, $3.2\left(\mathrm{~m}_{\mathrm{c}}, 3 \mathrm{H}\right), 3.68\left(\mathrm{~s}, 3 \mathrm{H}, \mathrm{OCH}_{3}\right), 3.79(\mathrm{~s}, 3 \mathrm{H}$, $\left.\mathrm{OCH}_{3}\right), 4.45[\mathrm{~d}, 1 \mathrm{H}, J(\mathrm{H} 5, \mathrm{H} 17) 1.19 \mathrm{~Hz}$, W-coupling, H 5], 5.51 [d, $1 \mathrm{H}, J(\mathrm{H} 18, \mathrm{H} 17) 8.88$ $\mathrm{Hz}, \mathrm{H} 18], 6.00\left(\mathrm{~m}_{\mathrm{c}}, 2 \mathrm{H}, \mathrm{H} 17\right.$ and $\left.-\mathrm{CH}=\mathrm{CH}_{2}\right)$, $6.30\left[\mathrm{~d}, 1 \mathrm{H}, J\left(\mathrm{E}-\mathrm{CH}=\mathrm{CH}_{2}-\mathrm{CH}=\mathrm{CH}_{2}\right) 16.82 \mathrm{~Hz}\right.$, $\left.-\mathrm{CH}=\mathrm{CH}_{2}\right], 6.52[\mathrm{~d}, 1 \mathrm{H}, J(\mathrm{H} 1, \mathrm{H} 2) 8.20 \mathrm{~Hz}, \mathrm{H} 1]$, $6.62(\mathrm{~d}, 1 \mathrm{H}, J(\mathrm{H} 2, \mathrm{H} 1) 8.07 \mathrm{~Hz}, \mathrm{H} 2], 6.79$ [dd, $1 \mathrm{H}, \quad J\left(\mathrm{E}-\mathrm{C} \underline{\mathrm{H}}=\mathrm{CH}_{2}-\mathrm{CH}=\mathrm{CH}_{2}\right) \quad 16.65 \quad \mathrm{~Hz}$, $\left.J\left(\mathrm{Z}-\mathrm{C} \underline{\mathrm{H}}=\mathrm{CH}_{2}-\mathrm{CH}=\mathrm{C}_{2}\right) 9.81 \mathrm{~Hz},-\mathrm{C} \underline{\mathrm{H}}=\mathrm{CH}_{2}\right)$. $-{ }^{13} \mathrm{C}$ NMR $\left(50.3 \mathrm{MHz}, \mathrm{CDCl}_{3}\right): \delta\left(\mathrm{C}_{\mathrm{p}}\right)=43.40$, $54.15,56.62\left(1 \times \mathrm{NCH}_{3}, 2 \times \mathrm{OCH}_{3}\right) ; \delta\left(\mathrm{C}_{\mathrm{s}}\right)=22.35$, $28.25,33.24,42.75,45.25$ (C 8, C 10, C 15, C 16, $\left.-\mathrm{CH}=\mathrm{CH}_{2}\right) ; \delta\left(\mathrm{C}_{\mathrm{t}}\right)=59.70,62.93,95.47(\mathrm{C} 5, \mathrm{C} 7$, C9), 113.71, 119.77, $125.11 \quad(\mathrm{C} 17, \mathrm{C} 18$, $\left.-\mathrm{C} H=\mathrm{CH}_{2}\right), 135.48,138.93(\mathrm{C} 1, \mathrm{C} 2) ; \delta\left(\mathrm{C}_{\mathrm{q}}\right)=$ $47.08,80.16,128.04$ (C6, C 13, C 14), 128.06, $133.65,142.50,147.61$ (C3, C4, C 11, C 12). EI-MS: $m / z(\%)=429(16)[\mathrm{M}]^{+} \cdot, 338(43)$.

$\mathrm{C}_{23} \mathrm{H}_{27} \mathrm{NSO}_{5}$ (429.54)

Calcd C 64.31 H 6.34 N 3.26 S 7.46\%, Found C 64.52 H 6.45 N 3.16 S 7.60\%.
4,5a-Epoxy-7 $\beta, 8$ a-bis (methoxycarbonyl)3,6-dimethoxy- $\mathrm{N}$-methyl-6a, $14 a$-endoetheno-isomorphinane (3a)

(-)-Thebaine (1) (200 mg, $0.64 \mathrm{mmol})$ and dimethyl fumarate $(365 \mathrm{mg}, 3.2 \mathrm{mmol})$ were heated under reflux in $30 \mathrm{ml}$ toluene for $4 \mathrm{~d}$. The mixture was then concentrated to $1 \mathrm{ml}$ and the residue obtained was purified by flash chromatography (ethyl acetate/petroleum, ether 3:1). Yield $140 \mathrm{mg}$ (48\%), m.p. $159-162{ }^{\circ} \mathrm{C}$ (ethyl acetate). $-{ }^{1} \mathrm{H}$ NMR $\left(200 \mathrm{MHz}, \mathrm{CDCl}_{3}\right): \delta=1.72[\mathrm{dd}, 1 \mathrm{H}$, $J(\mathrm{H} 15, \mathrm{H} 15) 13.53 \mathrm{~Hz}, J(\mathrm{H} 15, \mathrm{H} 16) 2.87 \mathrm{~Hz}$, $\mathrm{H} 15], 2.34\left(\mathrm{~s}, 3 \mathrm{H}, \mathrm{NCH}_{3}\right), 2.50\left(\mathrm{~m}_{\mathrm{c}}, 3 \mathrm{H}, \mathrm{H} 10\right.$, $2 \times \mathrm{H} 16), 2.82\left(\mathrm{~m}_{\mathrm{c}}, 1 \mathrm{H}, \mathrm{H} 15\right), 3.05[\mathrm{~d}, 1 \mathrm{H}, J(\mathrm{H} 7$, H 8) $5.61 \mathrm{~Hz}, \mathrm{H} 7), 3.22(\mathrm{~d}, 1 \mathrm{H}, J(\mathrm{H} 10, \mathrm{H} 10) 18.58$ $\mathrm{Hz}, \mathrm{H} 10), 3.54$ (s, $\left.3 \mathrm{H}, \mathrm{OCH}_{3}\right), 3.57$ [d, $1 \mathrm{H}, J(\mathrm{H} 9$, $\mathrm{H}$ 10) $6.79 \mathrm{~Hz}, \mathrm{H} 9$ ], 3.68 (s, $\left.3 \mathrm{H}, \mathrm{COOCH}_{3}\right), 3.77$ $\left(\mathrm{s}, 3 \mathrm{H}, \mathrm{OCH}_{3}\right), 3.80\left(\mathrm{~s}, 3 \mathrm{H}, \mathrm{OCH}_{3}\right), 4.16[\mathrm{~d}, 1 \mathrm{H}$, $J(\mathrm{H} 8, \mathrm{H} 7) 5.57 \mathrm{~Hz}, \mathrm{H} 8], 4.93[\mathrm{~d}, 1 \mathrm{H}, J(\mathrm{H} 5, \mathrm{H} 17)$ $1.04 \mathrm{~Hz}, \mathrm{~W}$-coupling, H 5)] $5.49[\mathrm{~d}, 1 \mathrm{H}, J(\mathrm{H} 18$, H 17) $8.88 \mathrm{~Hz}, \mathrm{H} 18], 6.06$ [dd, $1 \mathrm{H}, J(\mathrm{H} 17, \mathrm{H} 18)$ $8.94 \mathrm{~Hz}, J(\mathrm{H} 17, \mathrm{H} 5) 1.20 \mathrm{~Hz}, \mathrm{~W}$-coupling, H 17], $6.51[\mathrm{~d}, 1 \mathrm{H}, J(\mathrm{H} 2, \mathrm{H} 1) 8.14 \mathrm{~Hz}, \mathrm{H} 2], 6.62[\mathrm{~d}, 1 \mathrm{H}$, $J(\mathrm{H} 1, \mathrm{H} 2) 8.14 \mathrm{~Hz}, \mathrm{H} 1] .-{ }^{13} \mathrm{C} \mathrm{NMR}(50.3 \mathrm{MHz}$, $\left.\mathrm{CDCl}_{3}\right): \delta\left(\mathrm{C}_{\mathrm{p}}\right)=43.81,44.62,50.01,52.28,52.70$ $\left(1 \times \mathrm{NCH}_{3}, 4 \times \mathrm{OCH}_{3}\right) ; \delta\left(\mathrm{C}_{\mathrm{s}}\right)=22.95,30.88,45.55$ $(\mathrm{C} 10, \mathrm{C} 15, \mathrm{C} 16) ; \delta\left(\mathrm{C}_{\mathrm{t}}\right)=52.87,57.17,58.50$, 92.37 (C 5, C 7, C 8, C9), 114.43, 119.85 (C 17, $\mathrm{C} 18), 127.25,136.05(\mathrm{C} 1, \mathrm{C} 2) ; \delta\left(\mathrm{C}_{9}\right)=46.04$, $48.10,81.65$ (C6, C 13, C 14), 128.27, 135.06, $142.58,148.18$ (C 3, C4, C 11, C 12), 173.14, 173.74 $(2 \times \mathrm{CO}) .-$ EI-MS: $m / z(\%)=455(69)[\mathrm{M}]^{+}, 311$ (13).

$$
\begin{aligned}
& \mathrm{C}_{25} \mathrm{H}_{29} \mathrm{NO}_{7}(455.52) \\
& \text { Calcd C } 65.92 \text { H } 6.42 \text { N 3.07\%, } \\
& \text { Found C } 65.96 \text { H } 6.37 \text { N 3.16\%. }
\end{aligned}
$$

\section{4,5a-Epoxy-7 $\beta, 8$ a-bis (ethoxycarbonyl)- 3,6-dimethoxy- $N$-methyl-6a, 14 a-endo- etheno-isomorphinane (3b)}

From (-)-thebaine (1) (200 mg, $0.64 \mathrm{mmol})$ and diethyl fumarate $(551 \mathrm{mg}, 3.2 \mathrm{mmol})$ following the procedure described above for compound $3 \mathbf{a}$. Yield $160 \mathrm{mg}(52 \%)$, m.p. $108-110^{\circ} \mathrm{C}$ (ethyl acetate). $-{ }^{1} \mathrm{H} \mathrm{NMR}\left(200 \mathrm{MHz}, \mathrm{CDCl}_{3}\right): \delta=1.27\left(\mathrm{~m}_{\mathrm{c}}\right.$, $\left.6 \mathrm{H}, 2 \times \mathrm{OCH}_{2} \mathrm{CH}_{3}\right), 1.70[\mathrm{dd}, 1 \mathrm{H}, J(\mathrm{H} 15, \mathrm{H} 15)$ $13.65 \mathrm{~Hz}, J(\mathrm{H} 15, \mathrm{H} 16) 2.71 \mathrm{~Hz}, \mathrm{H} 15], 2.34$ (s, $3 \mathrm{H}$, $\left.\mathrm{NCH}_{3}\right), 2.50\left(\mathrm{~m}_{\mathrm{c}}, 3 \mathrm{H}, \mathrm{H} 10,2 \times \mathrm{H} 16\right), 2.84\left(\mathrm{~m}_{\mathrm{c}}\right.$, $1 \mathrm{H}, \mathrm{H} 15), 3.02$ [d, $1 \mathrm{H}, J(\mathrm{H} 7, \mathrm{H} 8) 5.71 \mathrm{~Hz}, \mathrm{H} 7$ ], 3.21 [d, $1 \mathrm{H}, J(\mathrm{H} 10, \mathrm{H} 10) 18.58 \mathrm{~Hz}, \mathrm{H} 10], 3.54$ (s, $\left.3 \mathrm{H}, \mathrm{OCH}_{3}\right), 3.61[\mathrm{~d}, 1 \mathrm{H}, J(\mathrm{H} 9, \mathrm{H} 10) 6.52 \mathrm{~Hz}$, $\mathrm{H} 9], 3.80\left(\mathrm{~s}, 3 \mathrm{H}, \mathrm{OCH}_{3}\right), 4.20\left(\mathrm{~m}_{\mathrm{c}}, 5 \mathrm{H}, \mathrm{H} 8\right.$, $\left.2 \times \mathrm{OC}_{2} \mathrm{CH}_{3}\right), 4.94$ [d, $1 \mathrm{H}, J(\mathrm{H} 5, \mathrm{H} 17) 1.40 \mathrm{~Hz}$, 
W-coupling, H 5], 5.49 [d, $1 \mathrm{H}, J(\mathrm{H} 18 ; \mathrm{H} 17) 8.85$ $\mathrm{Hz}, \mathrm{H} 18$ ] 6.05 [dd, $1 \mathrm{H}, J(\mathrm{H} 17, \mathrm{H} 18) 8.87 \mathrm{~Hz}$, $J(\mathrm{H} 17, \mathrm{H} 5) 1.43 \mathrm{~Hz}, \mathrm{H} 17], 6.51[\mathrm{~d}, 1 \mathrm{H}, J(\mathrm{H} 1$, H2) $8.19 \mathrm{~Hz}, \mathrm{H} 1], 6.62$ [d, $1 \mathrm{H}, J(\mathrm{H} 2, \mathrm{H} 1) 8.11 \mathrm{~Hz}$, $\mathrm{H} 2] .-{ }^{13} \mathrm{C} \mathrm{NMR}\left(50.3 \mathrm{MHz}, \mathrm{CDCl}_{3}\right): \delta\left(\mathrm{C}_{\mathrm{p}}\right)=$ $14.20,14.24,43.44,44.16,50.14\left(\mathrm{NCH}_{3}, 2 \times \mathrm{OCH}_{3}\right.$, $\left.2 \times \mathrm{OCH}_{2} \mathrm{CH}_{3}\right) ; \delta\left(\mathrm{C}_{\mathrm{s}}\right)=22.46,30.19,45.11,60.70$, $61.21\left(\mathrm{C} 10, \mathrm{C} 15, \mathrm{C} 16,2 \times \mathrm{OCH}_{2} \mathrm{CH}_{3}\right) ; \delta\left(\mathrm{C}_{\mathrm{t}}\right)=$ $52.71,56.86,57.97,91.99$ (C 5, C 7, C 8, C9), $114.15,119.24$ (C 17, C 18), 127.07, 135.35 (C 1, $\mathrm{C} 2) ; \delta\left(\mathrm{C}_{\mathrm{q}}\right)=45.47,47.60,81.13(\mathrm{C} 6, \mathrm{C} 13, \mathrm{C} 14)$, $127.54,134.56,142.11,147.73(\mathrm{C} 3, \mathrm{C} 4, \mathrm{C} 11$, C 12), 172.31, $173.05(2 \times \mathrm{CO})$. - EI-MS: $m / z(\%)$ $=483(100)[\mathrm{M}]^{+} \cdot, 311(21)$.

Analysis $\mathrm{C}_{27} \mathrm{H}_{33} \mathrm{NO}_{7}(483.57)$

Calcd C 67.06 H 6.88 N 2.90\%,

Found C 67.12 H 6.75 N 3.02\%.

\section{4,5 a-Epoxy-7 a,8 a-bis (methoxycarbonyl)- 3,6-dimethoxy- $N$-methyl-6 a, 14 a-endo- etheno-isomorphinane (4)}

(-)-Thebaine (1) (200 mg, $0.64 \mathrm{mmol})$ and dimethyl maleate $(553.5 \mathrm{mg}, 3.84 \mathrm{mmol})$ were heated under reflux in $30 \mathrm{ml}$ toluene for $4 \mathrm{~d}$. The mixture was then concentrated to $1 \mathrm{ml}$ and stored for $24 \mathrm{~h}$ at $6{ }^{\circ} \mathrm{C}$. The formed solid product was crystallized several times from methanol. Yield $80 \mathrm{mg}(28 \%)$, m.p. $188-190{ }^{\circ} \mathrm{C}, 189-190{ }^{\circ} \mathrm{C}[11 \mathrm{a}] .-{ }^{1} \mathrm{H}$ NMR (200 MHz, $\left.\mathrm{CD}_{2} \mathrm{Cl}_{2}\right): \delta=1.83\left(\mathrm{~m}_{\mathrm{c}}, 1 \mathrm{H}, \mathrm{H} 15\right), 1.95$ $\left(\mathrm{m}_{\mathrm{c}}, 1 \mathrm{H}, \mathrm{H} 15\right), 2.30\left(\mathrm{~s}, 3 \mathrm{H}, \mathrm{NCH}_{3}\right), 2.38\left(\mathrm{~m}_{\mathrm{c}}, 1 \mathrm{H}\right.$, H 16), $2.49\left(\mathrm{~m}_{\mathrm{c}}, 2 \mathrm{H}, \mathrm{H} 10, \mathrm{H} 16\right), 3.19[\mathrm{~d}, 1 \mathrm{H}$, $J(\mathrm{H} 10, \mathrm{H} 10) 18.66 \mathrm{~Hz}, \mathrm{H} 10], 3.36$ [d, $1 \mathrm{H}, J(\mathrm{H} 7$, H 8) $11.12 \mathrm{~Hz}, \mathrm{H} \mathrm{17]}, 3.50$ (s, $\left.3 \mathrm{H}, \mathrm{OCH}_{3}\right), 3.53$ (s, $\left.3 \mathrm{H}, \mathrm{OCH}_{3}\right), 3.61\left(\mathrm{~s}, 3 \mathrm{H}, \mathrm{OCH}_{3}\right), 3.74[\mathrm{~d}, 1 \mathrm{H}$, $J(\mathrm{H} 9, \mathrm{H} 10) 6.62 \mathrm{~Hz}, \mathrm{H} 9], 3.78\left(\mathrm{~s}, 3 \mathrm{H}, \mathrm{OCH}_{3}\right)$, $4.14(\mathrm{~d}, 1 \mathrm{H}, J(\mathrm{H} 8, \mathrm{H} 7) 11.14 \mathrm{~Hz}, \mathrm{H} 8], 4.59$ [d, $1 \mathrm{H}, J(\mathrm{H} 5, \mathrm{H} 17) 0.91 \mathrm{~Hz}$, W-coupling, H 5], 5.77 [d, $1 \mathrm{H}, J(\mathrm{H} \mathrm{18}, \mathrm{H} 17) 8.85 \mathrm{~Hz}, \mathrm{H} 18], 5.88$ [d, $1 \mathrm{H}$, $J(\mathrm{H} 17, \mathrm{H} 18) 8.83 \mathrm{~Hz}, \mathrm{H} 17], 6.57$ [d, $1 \mathrm{H}, J(\mathrm{H} 1$, H2) $8.17 \mathrm{~Hz}, \mathrm{H} 1], 6.63$ [d, $1 \mathrm{H}, J(\mathrm{H} 2, \mathrm{H} 1) 8.18 \mathrm{~Hz}$, $\mathrm{H} 2] .-{ }^{13} \mathrm{C} \mathrm{NMR}\left(50.3 \mathrm{MHz}, \mathrm{CD}_{2} \mathrm{Cl}_{2}\right): \delta\left(\mathrm{C}_{\mathrm{p}}\right)=$ $43.75, \quad 46.20,49.11, \quad 51.63, \quad 52.10 \quad\left(1 \times \mathrm{NCH}_{3}\right.$, $\left.4 \times \mathrm{OCH}_{3}\right) ; \delta\left(\mathrm{C}_{\mathrm{s}}\right)=22.60,34.04,45.59(\mathrm{C} \mathrm{10}, \mathrm{C} 15$, $\mathrm{C} 16) ; \delta\left(\mathrm{C}_{\mathrm{t}}\right)=52.68,56.93,58.24,92.97(\mathrm{C} 5, \mathrm{C} 7$, C 8, C9), 114.16, 120.05 (C 17, C 18), 126.41, $135.08(\mathrm{C} 1, \mathrm{C} 2) ; \delta\left(\mathrm{C}_{\mathrm{q}}\right)=44.66,47.77,81.04(\mathrm{C} 6$, C 13, C 14), 128.73, 133.72, 142.30, 148.30 (C 3, C4, C11, C 12), 171.43, $171.56(2 \times \mathrm{CO}) .-$ EI-MS: $m / z(\%)=455(100)[\mathrm{M}]^{+} \cdot, 311(23)$.

Analysis $\mathrm{C}_{25} \mathrm{H}_{29} \mathrm{NO}_{7}$ (455.52) Calcd C 65.92 H 6.42 N 3.07\%, Found C 65.81 H 6.38 N 3.21\%.
8,17a-Epoxy-9,16-dimethoxy-5-methyl$5,6,7,7$ a $12,14 \beta$-hexahydro-7 a $\alpha, 14 \alpha-[1]$ propenobenzo[b]naphtho[2,1-e]azocine (5)

Anthranilic acid $(950 \mathrm{mg}, 6.92 \mathrm{mmol})$ and trichloroacetic acid $(8.4 \mathrm{mg}, 0.05 \mathrm{mmol})$ were suspended in $20 \mathrm{ml}$ tetrahydrofuran and cooled to $0{ }^{\circ} \mathrm{C}$. To this mixture in over a period of $2 \mathrm{~min}$ was added isoamyl nitrite $(1.6 \mathrm{ml}, 11.8 \mathrm{mmol})$ at same temperature. After the mixture had been stirred at $20{ }^{\circ} \mathrm{C}$ for $1.5 \mathrm{~h}$ the precipitate was separated and purified by washing with tetrahydrofuran. The thus obtained $o$-benzene-diazonium carboxylate was suspended in dichloromethane. The resultant suspension was added in small portions to a refluxing solution of (-)-thebaine (1) $(388.75 \mathrm{mg}, 1.25$ $\mathrm{mmol}$ ) in $25 \mathrm{ml}$ dichloromethane over a period of $30 \mathrm{~min}$. After being heated under reflux for $30 \mathrm{~min}$, the mixture was concentrated carefully under reduced pressure and the residue obtained purified by flash chromatography (ethyl acetate/petroleum ether, $3: 1$ ). Yield $30 \mathrm{mg}(6 \%)$, m.p. $186{ }^{\circ} \mathrm{C}$ (ethyl acetate). $-{ }^{1} \mathrm{H} \mathrm{NMR}\left(400 \mathrm{MHz}, \mathrm{CD}_{2} \mathrm{Cl}_{2}\right): \delta=1.59$ [dd, $1 \mathrm{H}, J(\mathrm{H} 7, \mathrm{H} 7) 13.94 \mathrm{~Hz}, J(\mathrm{H} 7, \mathrm{H} 6) 4.38 \mathrm{~Hz}$, H 7], $2.33\left(\mathrm{~m}_{\mathrm{c}}, 1 \mathrm{H}, \mathrm{H} 6\right), 2.75\left(\mathrm{~s}, 3 \mathrm{H}, \mathrm{NCH}_{3}\right), 2.94$ $\left(\mathrm{m}_{\mathrm{c}}, 2 \mathrm{H}, \mathrm{H} 6, \mathrm{H} 7\right), 3.22\left(\mathrm{~m}_{\mathrm{c}}, 2 \mathrm{H}, 2 \times \mathrm{H} 12\right), 3.45$ (s, $\left.3 \mathrm{H}, \mathrm{OCH}_{3}\right), 3.84\left(\mathrm{~s}, 3 \mathrm{H}, \mathrm{OCH}_{3}\right), 4.21[\mathrm{~d}, 1 \mathrm{H}$, $J(\mathrm{H} 14, \mathrm{H} 15) 6.07 \mathrm{~Hz}, \mathrm{H} 14], 4.74$ [d, $1 \mathrm{H}, J(\mathrm{H} 15$, H 14) $6.14 \mathrm{~Hz}, \mathrm{H} 15], 5.43$ (s, 1 H, H 17), 6.14 [dd, $1 \mathrm{H}, J(\mathrm{H} 13, \mathrm{H} 12)=5.73, J(\mathrm{H} 13, \mathrm{H} 14) 2.59 \mathrm{~Hz}$, H 13], 6.66 [d, 1 H, J(H 11, H 10) $8.02 \mathrm{~Hz}, \mathrm{H} 10$ ], $6.69[\mathrm{~d}, 1 \mathrm{H}, J(\mathrm{H} 10, \mathrm{H} 11) 8.10 \mathrm{~Hz}, \mathrm{H} 10], 6.98\left(\mathrm{~m}_{\mathrm{c}}\right.$, $1 \mathrm{H}$, aromatic), $7.19\left(\mathrm{~m}_{\mathrm{c}}, 3 \mathrm{H}\right.$, aromatic). $-{ }^{13} \mathrm{C}$ NMR (50.3 MHz, $\left.\mathrm{CD}_{2} \mathrm{Cl}_{2}\right): \delta\left(\mathrm{C}_{\mathrm{p}}\right)=39.24,49.87$, $54.87\left(1 \times \mathrm{NCH}_{3}, 2 \times \mathrm{OCH}_{3}\right) ; \delta\left(\mathrm{C}_{\mathrm{s}}\right)=29.78,37.23$, $60.71(\mathrm{C} 6, \mathrm{C} 7, \mathrm{C} 12) ; \delta\left(\mathrm{C}_{\mathrm{t}}\right)=56.87,88.75(\mathrm{C} 14$, C 17), 100.64, 112.41 (C13, C 15), 119.15, 119.88, $123.65,125.91,127.79,131.43$ (C1, C 2, C3, C4, $\mathrm{C} 10, \mathrm{C} 11) ; \delta\left(\mathrm{C}_{\mathrm{q}}\right)=51.33(\mathrm{C} 7 \mathrm{a}), 128.79,136.13$ (C13a, C 16), 139.89, 143.03, 143.31, 145.03, $152.70,154.76$ (C4a, C 7b, C8, C9, C11a, C 14a). - EI-MS: $m / z(\%)=387(100)[\mathrm{M}]^{+}$.

Analysis $\mathrm{C}_{25} \mathrm{H}_{25} \mathrm{NO}_{3}$ (387.49) Calcd C 77.49 H 6.50 N $3.61 \%$, Found C 76.69 H 6.98 N 3.84\%.

We thank the Deutsche Forschungsgemeinschaft (Bonn) for financial support of this work and are also grateful to $\mathrm{H}$. Kolshorn, Institute of Organic Chemistry, University of Mainz, for the special NMR spectroscopic measurements. 
[1] K. W. Bentley, The Chemistry of the Morphine Alkaloids, Clarendon Press, pp. 209-301, Oxford (1954).

[2] K. W. Bentley, in R. H. F. Manske (ed.): The Alkaloids, Vol. 8, Academic Press, pp. 75-125 London, New York (1971).

[3] K. W. Bentley, D. G. Hardy, J. Am. Chem. Soc. 89, 3267 (1967).

[4] O. Hromatka, G. Sengstschmid, Monatsh. Chem. 102, 1022 (1971); R. Giger, R. Rubinstein, D. Ginsburg, Tetrahedron 29, 2387 (1973).

[5] J. Marton, Z. Szabo, S. Hosztafi, Liebigs Ann. Chem. 1993, 915.

[6] U. Pindur, D. Keilhofer, Liebigs Ann. Chem. 1993, 947.

[7] U. Pindur, G. Lutz, G. Fischer, D. Schollmeyer, W. Massa, L. Schröder, Tetrahedron 49, 2863 (1993).

[8] K. W. Bentley, J. W. Lewis, A. C. B. Smith, J. Chem. Soc., Perkin Trans. I 1972, 870; see also ref. [3].

[9] M. J. S. Dewar, E. G. Zoebisch, J. J. P. Stewart, J. Am. Chem. Soc. 107, 3902 (1985).

[10] I. Fleming, Frontier Orbitals and Organic Chemical Reactions, John Wiley \& Sons, New York (1976).

[11] O. Hromatka, G. Sengstschmid, K. Eichinger, Monatsh. Chem. 102, 1015 (1971); K. W. Bentley, A. F. Thomas, J. Chem. Soc., Perkin Trans. I 1985, 1863; R. Rubinstein, F. Haviv, D. Ginsburg Tetrahedron 30, 1201 (1974).

[12] J. Sauer, R. Sustmann, Angew. Chem. 92, 773 (1980); Angew. Chem., Int. Ed. Engl. 19, 779 (1980).

[13] H. Rapoport, P. Sheldrick, J. Am. Chem. Soc. 85, 1636 (1963).

[14] K. Hayakawa, J. Fujii, K. Kanematsu, J. Org. Chem. 48, 166 (1983).
[15] A. Singh, S. Archer, J. Org. Chem. 48, 173 (1983).

[16] L. Friedman, F. M. Logullo, J. Org. Chem. 34, 3089 (1969); F. M. Logullo, A. H. Seitz, L. Friedman, Org. Synth. 4, 54 (1973).

[17] T. L. Gilchrist, in S. Patai and Z. Rappoport (eds): The Chemistry of Functional Groups, Suppl. C, p. 383, John Wiley \& Sons, London (1983).

[18] For details of "quantitative" NOE analysis and relaxation time $\left(T_{1}\right)$ see: J. H. Noggle and R. E. Schirmer, The Nuclear Overhauser Effects, Chemical Applications, Academic Press, New York (1971); H. Günther, NMR-Spektroskopie, Georg Thieme Verlag, Stuttgart (1983); H. Friebolin, einund zweidimensionale NMR-Spektroskopie, VCH, Weinheim (1988).

[19] MMX force field calculations (a variant of MM2 method) see: J. J. Gajewski, K. E. Gilbert, J. McKelvey, Adv. Molec. Mod. 2, 65 (1990); the program packet PCMODEL 3.2 from Serena Software, Bloomington, IN, was used.

[20] G. M. Sheldrick, SHELXS-86, Program for Crystal Structure Determination, University of Göttingen (1986).

[21] G. M. Sheldrick, SHELXL-93, Program for Refinement of Crystal Structure, University of Göttingen (1993).

[22] G. M: Sheldrick, SHELX-76, Program for Crystal Structure Determination, University of Cambridge (1976).

[23] E. Keller, Program SCHAKAL, Version 92, University of Freiburg.

[24] M. Jaskolski, Program GEOM; Program to Calculate Geometrical Features of Molecules, University of Poznau, Poland (1982). 